\title{
ESTUDIO ETNOBOTÁNICO DEL MORTIÑO (Vaccinium floribundum) COMO ALIMENTO ANCESTRAL Y POTENCIAL ALIMENTO FUNCIONAL
}

\author{
ETHNOBOTANICAL STUDY OF THE MORTIÑO (Vaccinium floribundum) AS \\ ANCESTRAL AND POTENTIALLY FUNCTIONAL MEAL \\ Pablo Coba Santamaría, Daniel Coronel, Karla Verdugo, María Fernanda \\ Paredes, Elizabeth Yugsi y Laura Huachi
}

Centro de Investigación y Valoración de la Biodiversidad CIVABI. Universidad Politécnica Salesiana. Av. 12 de Octubre N24-22 y Wilson. Bloque B. Tercer Piso. Telfs. 593023962800.

Autor para correspondencia: pcoba@ups.edu.ec

Manuscrito recibido el 20 de noviembre de 2012. Aceptado, tras revisión, el 14 de diciembre de 2012.

\section{Resumen}

El mortiño es un arbusto silvestre endémico de Ecuador y Colombia. Posee una baya de agradable sabor, frutos de especies emparentadas al él eran consumidos desde antes de la Conquista, elevándola a fruta ceremonial. Tradición que se ha derivado a la celebración del Día de los Difuntos en Ecuador. Su propiedad relevante es poseer gran cantidad de polifenoles asistida por el contenido de azúcares, fibra, lípidos, minerales y vitaminas, proantocianidinas, antocianidinas, flavonoides se los utiliza como colorantes alimentarios; así como flavonoides, taninos, ácidos poli fenólicos tienen actividad antioxidante, coadyuvante en la microcirculación retiniana y antiagregación plaquetaria, microcirculación en general, reducción en la insuficiencia venosa crónica, considerado como coadyuvante nutrimental.

Palabras claves: Mortiño, Vaccinium floribundum, capacidad antioxidante.

\footnotetext{
Abstract

Mortiño is a native wild plant from Ecuador and Colombia. With an edible and tasty berry, mortiño has been consumed since prehispanic ages with a ceremonial significance that is reflected nowadays in the celebration of Día de los difuntos (All Souls' day). The relevant properties are the high content of polyphenols due to the presence of sugars, fibers, lipids, minerals, vitamins proanthocyanidins, anthocyanidins and flavonoids that permits their use as food colorants. Compounds as flavonoids, tannins and poli-polyphenic acids are recognized as antioxidants and promote the retinal and general micro circulation, platelet antiaggregation as well as the reduction of chronic venous insufficiency, potentially suggesting mortiño as a nutritional supplement.

Keywords: Mortiño, Vaccinium floribundum, antioxydants.

Forma sugerida de citar: Coba, P., D. Coronel, K. Verdugo, M. Paredes, E. Yugsi y L. Huachi. 2012. Estudio etnobotánico del mortiño (Vaccinium floribundum) como alimento ancestral y potencial alimento funcional. La Granja. Vol. 16(2): 5-13. ISSN: 1390-3799.
} 


\section{Introducción}

La región de los Andes es uno de los mayores centros de domesticación de plantas del mundo (Vavilov, 1951), fue escenario de civilizaciones que desarrollaron una agricultura autóctona y tradicional con un gran número de especies de plantas nativas dentro de la franja altitudinal que oscila entre los 2500 y 4300 msnm.

El cultivo de estas especies fue la base alimenticia de grandes poblaciones andinas hasta la llegada de los españoles, cuando estas especies nativas fueron reemplazadas por especies exóticas traídas de Europa. Pese a una muy marcada invasión vegetal, algunas especies endémicas no fueron erradicadas de los suelos andinos ni de la alimentación de sus pobladores. En la actualidad, a pesar de la predominancia de los cultivos introducidos ya sean granos, hortalizas o frutos todavía existen cultivos tradicionales en los Andes y valles interandinos que aún siguen siendo la base de la alimentación de comunidades indígenas.

El Departamento de Agricultura de los Estados Unidos ha registrado una sola especie, Vaccinium floribundum en Ecuador (Ramírez y Williams, 2003), sin embargo, datos del Herbario de la Pontificia Universidad Católica del Ecuador, indican que se encuentran registradas tres especies de mortiño, la cuales son: Vaccinium distichum, Vaccinium crenatum, y Vaccinium floribundum, siendo la especie más común Vaccinium floribundum.

El mortiño (Vaccinium floribundum), de la familia Ericaceae, llamado también uva de monte, es una fruta nativa de los páramos ecuatorianos (Loján, 2003). En páramos ecuatorianos es considerada endémica y ha sido utilizada por sus habitantes desde tiempos inmemoriales principalmente en el Día de los Difuntos para la elaboración de la tradicional colada morada (ver Figura 1). En la actualidad aunque es poco común se lo emplea para consumo fresco así como en jugos, mermeladas y dulces. Sus frutos tienen contenidos importantes de azúcares, minerales, antioxidantes, vitaminas del complejo B, C y minerales como potasio, calcio, y fósforo (Morales, 2011). Por sus propiedades físico químicas presenta la ventaja de ser refrigerado sin alteración de sus características organolépticas y nutricionales, ni variaciones en peso o volumen, para la elaboración posterior de cualquier producto con valor agregado, lo cual facilita poder mantener un mercado permanente, aun fuera de las épocas de cosecha.

En décadas anteriores este producto tenía importancia dentro de la alimentación ecuatoriana y era de fácil adquisición en los campos de la Sierra, pero con el pasar de los años su consumo ha disminuido y la planta también ha comenzado a desaparecer, debido al limitado conocimiento acerca de sus beneficios y la dificultad para su propagación. Estudios han mencionado que este fruto es de crecimiento silvestre y de una pequeña producción anual, considerando la posibilidad de reproducción in-vitro, por multiplicación vegetativa con medianos resultados (Torres y Trujillo, 2010; Freire, 2004).

Para desarrollar una nueva brecha agroindustrial es necesario conocer los aspectos botánicos y sus aplicaciones dentro de la cultura popular, como su uso actual en el campo médico, industrial y culinario.

\section{Enfoque botánico y distribu- ción del mortiño en el Ecuador}

El mortiño pertenece al Reino Plantae, Filo Magnoliophyta, Clase Magnoliopsida, Orden Ericales, Familia Ericaceae, Nombre Científico Vaccinium floribundum, Sinonimia Vaccinium mortinia (Freire, 2004).

Es un arbusto ramificado cuya altura llega hasta $2,5 \mathrm{~m}$, de hojas muy pequeñas con el margen aserrado o crenado, nervación pinnada, flores de menos de $1 \mathrm{~cm}$, solitarias o en racimos; tubo del cáliz articulado o no con el pedicelo, hipanto globoso, 5 lóbulos lanceolados; corola urceolada, blanca o rosada, con 5 lóbulos reflexos, estambres de 8 a 10, del mismo largo que el tubo de la corola, filamentos libres, anteras con túbulos cortos, dehiscencia apical poricida; ovario ínfero, 5 locular, estilo ligeramente más largo que el tubo de la corola. El fruto es una baya esférica de 5 a $8 \mathrm{~mm}$ de diámetro de color azul y azul oscuro, lisa, a veces glauca (Jorgensen et al., 1995), (ver Figura 2).

El mortiño pertenece a la familia de las Ericaceae, es uno de los componentes florísticos más sobresalientes del neotrópico, comunes en las zonas 


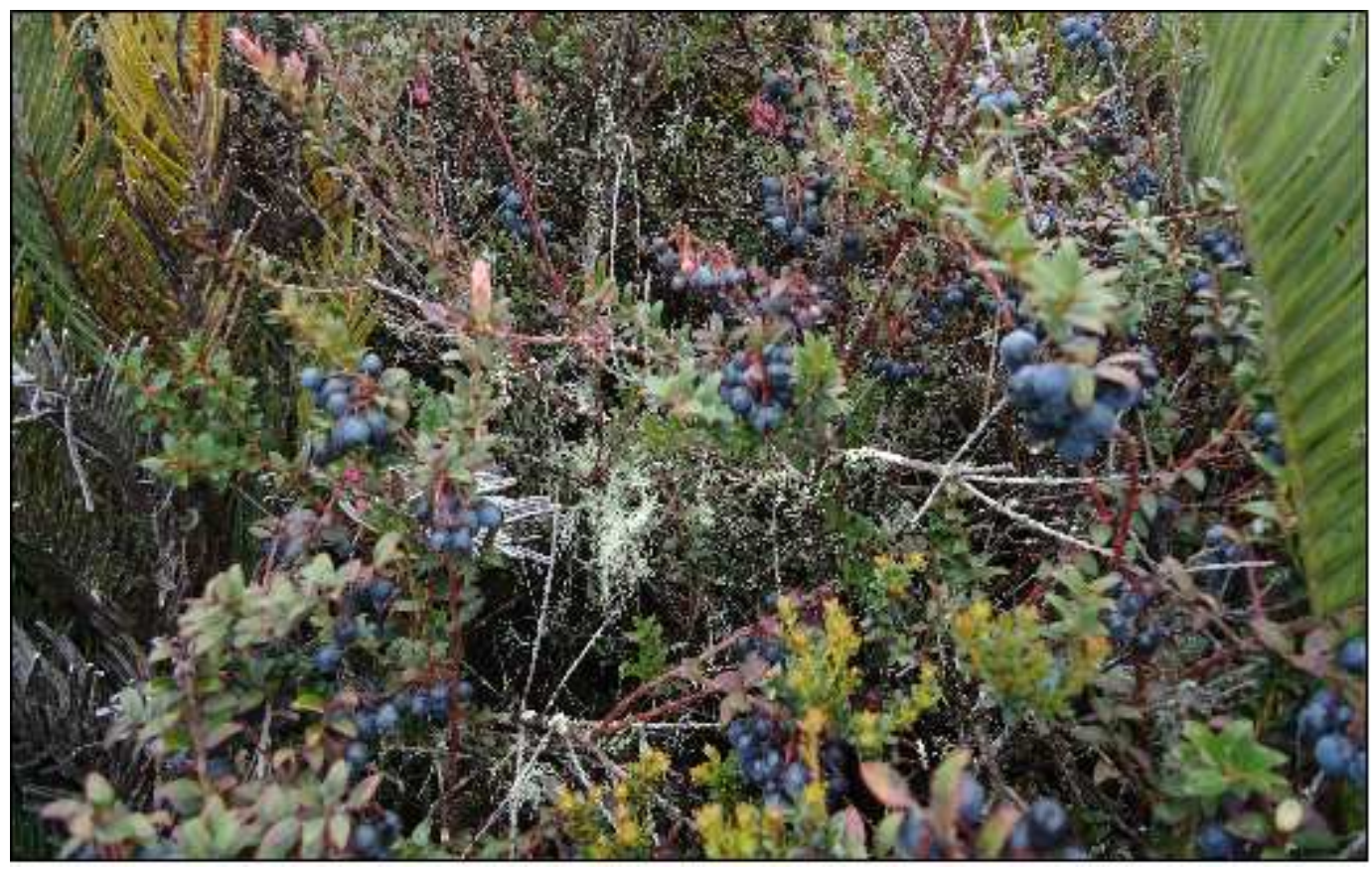

Figura 1. Recolección de mortiño en la Reserva de Cayambe-Coca-Cayapas (2012).

montañosas de noroccidente de Suramérica, especialmente en la vertiente pacífica de los Andes (Luteyn y Silva, 1999).

Posee 4500 especies a nivel mundial y alrededor de 900 en América Tropical (Smith et al., 2004), estas especies presentan hábitos variados, desde arbustos epífitos o hemiepífitos hasta litófitos. Presenta una alta diversidad de géneros y especies, entre ellas Rhododendron (con más de mil especies), Vaccinium (con cerca de 450 especies), Cavendishia (con cerca de 130 especies) y Gaultheria (con cerca de 115 especies); por otra parte, tiene amplia distribución geográfica y abarcan todos los continentes a excepción de la Antártida. Los principales centros de diversidad se encuentran en las regiones montañosas del Neotrópico y de Papúa, Nueva Guinea (Kron et al., 2002).

Existe mucha diversidad de esta planta, que ha sido atribuida a los suelos arenosos, gumíferos, ricos en materia orgánica y tipos de vegetación, como consecuencia de la orografía de la zona (Gentry, 1988; Oviedo et al., 1992).

En Colombia se han reportado en este género $V$. meridionale, $V$. floribundum y $V$. corymbodendron. (Luteyn, 2002), con consumo de los frutos de los dos primeros (Romero, 1991).
En Estados Unidos se le conoce como arándano y el lingoberry que son versiones americanas y europeas del mortiño (Asturizaga et al., 2006).

Los nombres vulgares con los cuales se conoce al mortiño en Ecuador son: mortiño, uva de los Andes, manzanilla de cerro, raspadura quemada, uva de monte; en Colombia se lo conoce como agraz, y en Perú como macha macha, congama, pushgay.

El mortiño se encuentra distribuido en los Andes ecuatorianos al norte desde la provincia del Carchi hasta la provincia de Loja al sur (Jorgensen y LeónYánez, 1999). Esta especie crece en un amplio rango altitudinal desde los 1600 hasta los $3800 \mathrm{~m}$ de altitud, se desarrolla en climas templados y fríos, con temperaturas de 8 a $16^{\circ} \mathrm{C}$, en los bosques seco montano bajo y húmedo montano, en suelos húmedos $\mathrm{y}$ bien drenados (Bernal y Correa, 1990).

En el Ecuador Vaccinium floribundum es considerada una planta silvestre que crece en las parte altas de la cordillera desde los páramos del Ángel en el Carchi hasta Tambo en Cañar, además se conocen datos proporcionados por el Parque Nacional Cotopaxi que ubican a la zona de adaptación del mortiño desde los $1000 \mathrm{msnm}$, hasta los $4500 \mathrm{msnm}$, pero la realidad es que son pocos los páramos que poseen un número considerable de plantas, debido a la ex- 


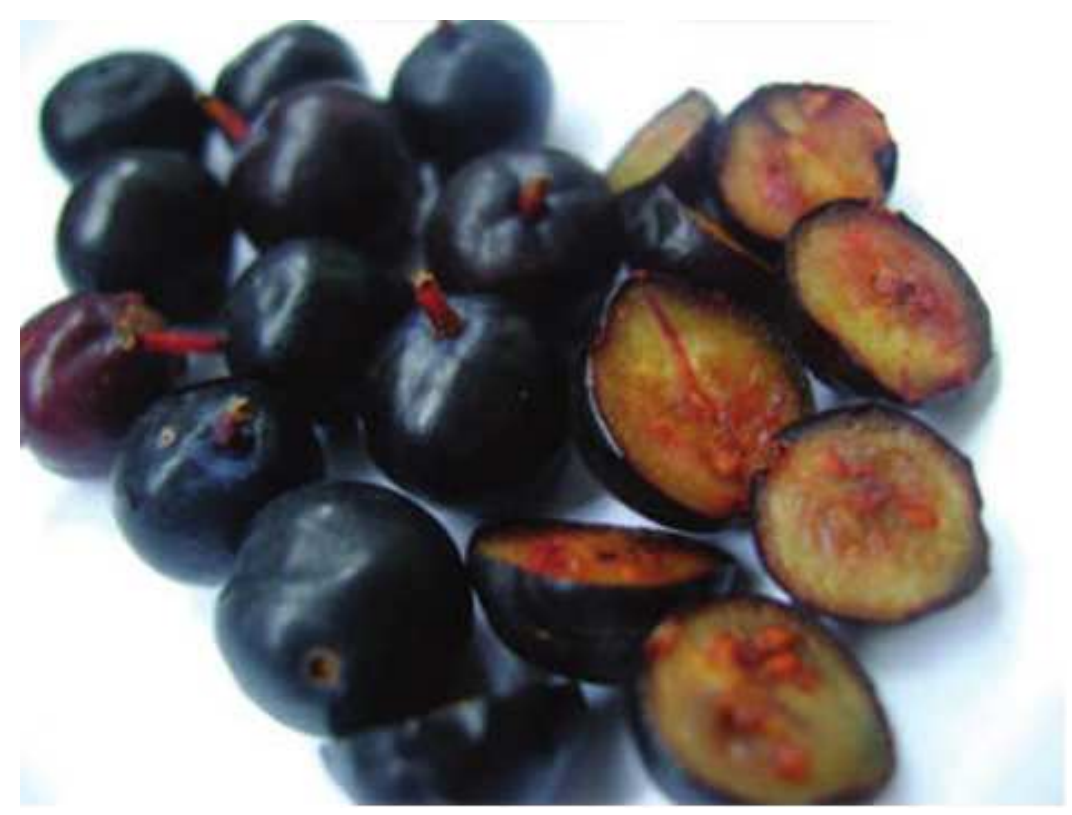

Figura 2. Fruto de mortiño (Vasco, 2009).

tensión de las áreas agrícolas que ha relegado a esta especie a zonas de páramo comprendidas entre los 3400 a 3500 hasta los 4500 msnm (MAGAP, 1988), como se muestras en la Figura 3.

En base a colectas realizadas Vaccinium floribundum se encuentra en la Sierra en las provincias de Carchi, Imbabura, Pichincha, Cotopaxi, Tungurahua, Bolivar, Chimborazo, Cañar, Azuay y Loja. De igual manera en base a colectas realizadas se indica que Vaccinium distichum y Vaccinium crenatum se encuentran localizados en las provincias del Azuay y Loja (Luteyn, 2002). Estrella, en su libro “El Pan de América" (1986), cita a Macleania ecuadoriensis "hualico", Macleania laurina Blake "Chaqui-lulu", Macleania popenoei "Joyapa", como bayas comestibles de características similares.

\section{El mortiño: valoración etnobo- tánica, florística y medioam- biental}

Tradicionalmente el mortiño se consume en un plato especial con miel de caña, especias y otros pedazos de frutas en el Día de los Difuntos, sin olvidar a la tradicional colada morada, un plato típico de la cultura popular. (Estrella, 1986). Antiguamente pocos le reconocían por su nombre y es probable que la mayor parte de la población urbana desconociera de su existencia, siendo de la familia del agraz o los arándanos.

Se considera al mortiño como un producto sagrado ya que es un fruto de características silvestres, por lo no requiere tratamiento alguno. Los campesinos utilizan este arbusto para calmar el reumatismo, fiebres y cólicos; se usa también para sanar la gripe, la borrachera y los dolencias del hígado y los riñones. Además para tratar dolencias pulmonares y la debilidad (CESA, 1993).

El mortiño es conocido como un arbusto ideal para fines ornamentales gracias a que posee hojas con características brillantes, lisas, de color granate $y$ rosadas en su juventud. Estas son usadas idealmente para adornar ambientes, ya que al ser un arbusto, con la poda, adquiere formas decorativas que suelen ser muy llamativas (Noboa, 2010; Torres y Trujillo, 2010).

Puede ser utilizado como tinte y de color duradero. Sus hojas se usan como forraje de borregos. Esta planta también sirve como combustible y regeneradora de sitios quemados empleándose en la reforestación de los páramos (Aguilar, 2009). 


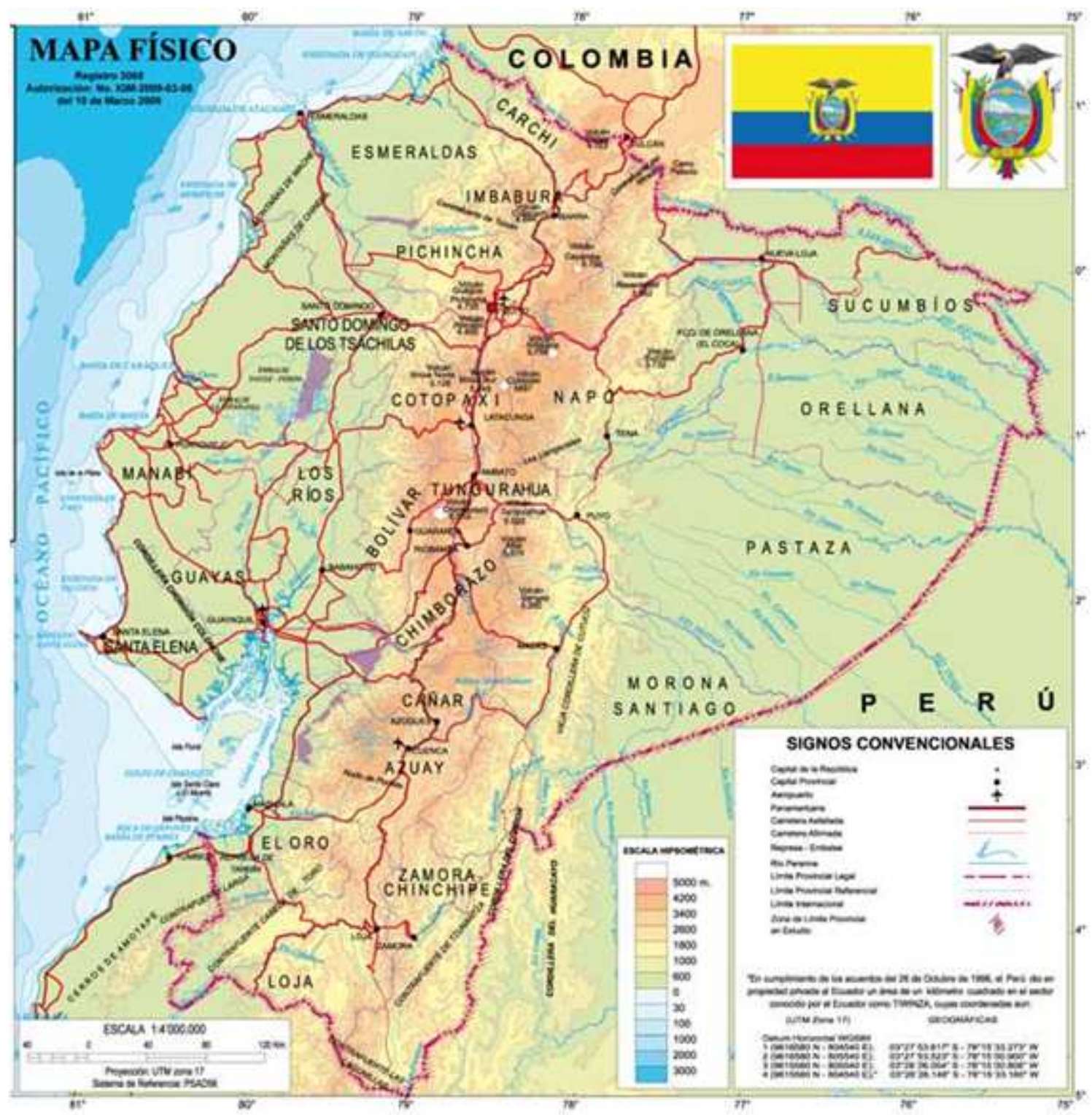

Figura 3. Zonificación del mortiño en Ecuador, (Aguilar, 2009), en base al mapa del Instituto Geográfico Militar IGMEcuador

\section{El mortiño: de alimento ances- tral a fuente de antioxidantes y vitaminas}

En la Región Andina, los frutos emparentados al mortiño eran consumidos desde antes de la Conquista (Cieza De León, 1962), en las cuales se describe la presencia de este fruto en los poblados andinos con el nombre de mortiños considerándola una fruta ceremonial, de la que se elaboraba una mazamorra para los muertos conocida como ayaapi (Estrella, 1986).

Asimismo, el fruto fresco, en su ambiente natural, es consumido por aves y animales autóctonos quienes dispersan las semillas en su entorno, el consumo no se limita a la alimentación animal sino también al ser humano por su exquisito sabor. En 
el Ecuador se reporta en la elaboración de postres, mermeladas, jugos, vinos, helados, mermeladas, jaleas, harina, frutos deshidratados, elaborados en panadería, bebidas no fermentadas, compotas y coladas (Fuentes, 2008).

La baya del mortiño consumida fresca aporta con agua $\sim 80 \%$; de proteína $\sim 0,7 \%$; grasa $\sim 1 \%$; carbohidratos totales $\sim 16,9 \%, 18,1 \%$; cenizas $\sim 0,4 \%$; fibra total $\sim 7,6 \%, 2,9 \%$; con un componente calórico de $\sim 84 \mathrm{kcal} / 100 \mathrm{~g} \mathrm{FF}, \sim 75 \mathrm{kcal} / 100 \mathrm{~g}$ FF (Vasco et al., 2009; Estrella, 1986); el proceso de cocción y el posterior consumo puede alterar la composición de los componentes nutricionales y nutracéuticos sin embargo no se han reportado estudios, tales datos se asemejan a especies emparentadas como el M. ecuadorensis Horold (Estrella, 1986).

Se ha evidenciado la presencia de minerales como Fe, $\mathrm{Cu}, \mathrm{Zn}, \mathrm{Ca}, \mathrm{Mg}$, K, conteniendo 0,64mg de $\mathrm{Fe} / 100 \mathrm{~g}$ FF y $17 \mathrm{~g} \mathrm{Ca} / 100 \mathrm{~g}$ FF, estos valores aportan a la dieta diaria recomendada de 10mg/día H; 18 mg/día M y 500 mg H\&M respectivamente (Badui, 2006).

Una característica nutricional relevante de las frutas es la cantidad de vitaminas que aportan al bienestar humano, a razón del mortiño se reporta la presencia de acido ascórbico $\sim 14 \mathrm{~g} / 100 \mathrm{~g}$ FF (Vasco et al., 2009), (USDA, 2010); 106,1 mg/100g FF (Vasco, 2009); $\beta$-carotenos $36 \mathrm{ug} / 100 \mathrm{~g}$ FF (Vasco et al., 2009); así como Tiamina $\sim 0,05 \mathrm{mg} / 100 \mathrm{~g}$ FF; Riboflavina $0,05 \mathrm{mg} / 100 \mathrm{~g}$ FF; Niacina $0,18 \mathrm{mg} / 100 \mathrm{~g} \mathrm{~F}$; ácido patoténico $\sim 0,09 \mathrm{mg} / 100 \mathrm{~g}$ FF (USDA, 2010).

Debido a su gran sabor, propiedades y cualidades nutricionales, estas bayas son un producto que cada vez es más consumido el mundo. Al tener un bajo contenido de calorías es favorable para su uso en dietas y además, por la presencia de compuestos fenólicos y fibra (Vasco, Rihinen, Ruales y KamalEldin, 2009), reduce el azúcar en la sangre. Por su alto contenido en vitaminas como la vitamina $C$, que es un excelente antioxidante celular y purificadora, mientras que vitamina A que fortalece a la vista

La Food and Drug Administration (FDA) de los Estados Unidos, según sus estándares, considera a estas bayas como frutos con bajo contenido de grasa y sodio, rico en fibras, vitaminas y libre de colesterol (USDA, 2010).

Se reportan, además, la presencia de otros componentes orgánicos como la glucosa, fructosa, ácido cítrico y málico responsables de su sabor así como compuestos considerados nutraceuticos con propiedades antioxidantes (Vasco et al., 2009; USDA, 2010).

Sin descartar que en la planta de mortiño sus partes tales como las hojas contienen flavonoides derivados de la quercetina (rutósido y avicularina), taninos catéquicos (6-10\%), ácidos triterpénicos (ursólico, oleanólico), iridoides, ácidos fenólicos, leucoantocianidinas y cromo (9,0 ppm) (Vasco et al., 2009).

El fruto al ser de color negro evidencia la alta concentración de antocianidinas como polifenoles de estos se han reportado la presencia de acido gálico y sus esteres, derivados del ácido vainillinico e hidroxibenzoico, proantocianidinas, quercetina, miricetina derivados del acido clorogénico e hidroxicinámico, antocianinas que eviencian una capacidad antioxidante de $1200 \mathrm{mg}$ Trolox/100g FF (23). Sin embargo se reportan (1302 mg Trolox/100) (23) en bayas andinas emparentadas, no obstante esta propiedad la poseen los Vaccinium; V. corymbosum L. hybrids (200-675 mg Trolox/100g FF) (Vasco, 2009).

La característica del fruto se ha demostrado que se debe a un proceso de estrés oxidativo en el cual se demuestra dos estadios para el $V$. meridionale Sw. (INIAP, 2006), demostrando que la fruta madura es la que posee los valores más altos de fenoles totales a los 143 días de formación

Algunos autores evidencian la consideración del fruto como medicinal ya que al ser consumido crudo ayuda a restablecer los niveles normales de azúcar en la sangre y al consumir el fruto cocido trata problemas de hipoglicemia, gripe y diabetes. También sirve para problemas digestivos, vasculares, entre otros (Aguilar, 2009), información etnobotánica cita que el fruto cocido es útil para el tratamiento de los diabéticos (Ríos, Koziol, Borgtoft Pedersen y Granda, 2007).

\section{El Mortiño Como Alimento Funcional}

La tendencia mundial respecto a la innovación de los alimentos está considerando la inclusión de sustancias de origen natural extraídas de una fuente vegetal, que por medio de la tecnología alimentaria se la incorpora; a la matriz del producto, considerando sus características organolépticas como el color y la textura. Ciertos autores consideran a estos ingredientes como "nutracéuticos", término que

LA GRANJA, Revista de Ciencias de la Vida, 16(2) 2012: 5-13. (C) 2012, Universidad Politécnica Salesiana, Ecuador. 
esta acuñado en simonía de los "fitoingredientes", sin embargo organismos de control como el FDA de los Estados Unidos considera o define a estas sustancias como "suplementos dietéticos" no obstante estos ingredientes pueden ser comercializados solos o incorporados. Dichos principios son sustancias que han demostrado una acción farmacológica en fitomedicina o que contribuyen a la salud y el buen funcionamiento del organismo, en el mercado ecuatoriano podemos encontrar una amplia gama de productos como el yogurt con fibra, aceite con $\beta$-carotenos etc.

Además, algunos de estos compuestos químicos se los ha utilizado como aditivos alimentarios como colorantes ya que son sustancias coloreadas por que contienen antocianócidos. Palabra compuesta derivada de cianos que significa azul. Lo que explica y ratifica la presencia de estos compuestos polifenóli$\cos$

Estos compuestos están en la lista de colorantes permitidos en la industria de alimentos normados e identificados como colorantes naturales E163 (CEE, 2009). Las antocianidinas y antocianócidos es una familia fitoquímica con gran variabilidad presentes en flores, frutos y otros órganos expuestos a la luz solar, en la familia Ericaceae, el mortiño presenta un pariente cercano que el arándano Vaccinium myrtillus que en su composición química presenta en su baya una composición de antocianidinas y antocianósidos del 0,5\% (Alonso, 2004), similar a la presencia en el mortiño, donde la caracterización fitoquímica demuestra la presencia de delfinidina, cianidina, petunidina, peonidina, malvidina y 3-glucosildelfinidina (Vasco, Rihinen, Ruales y Kamal-Eldin, 2009).

Estos compuestos han demostrado tener actividades en beneficio de la salud humana como actividad antioxidante (Salvayre, Braquet, Perruchot, y Douste-Blazy, 1982), coadyuvante en la microcirculación retiniana y antiagregación plaquetaria (Jayle, Aubry, Gavini, Braccini y De La Baume, 1965), (Belleoud, Leluan y Boyer, 1967), (Zavarise, 1968), microcirculación en general (Mian, Curri, Lietti, y Bombardelli, 1977), reducción en la insuficiencia venosa crónica (Grismondi, 1980), reparación tisular cuando se administra tópicamente (Mian, Curri, Lietti, y Bombardelli, 1977), reductor de la proliferación de depósitos de lípidos y calcio sobre la capa intima arterial, pero sin modificar los niveles de colesterol plasmático (Kaddar, Robert, Miskulin, Tixier, Brechemier, 1979).

Las antocianidinas y antocianosidos no han reportado en estudios clínicos efectos adversos y tóxicos; la DL50 reportada en ratas fue de $2000 \mathrm{mg} / \mathrm{kg}$; en perros se reportó de $3000 \mathrm{mg} / \mathrm{kg}$ de peso (Enandi, 1987). El mortiño se recomienda en caso de várices, flebitis y hemorroides, pues sus componentes fortifican las paredes de los vasos capilares. La infusión de las hojas secas ayuda a disminuir la glucosa en la sangre y tres vasos diarios de jugo reducen el riesgo de enfermedades (Quingalombo, 2010).

En Estados Unidos y Europa el arándano es una fruta cotizada y base de diversos productos alimenticios atribuidos a frutos del bosque; diversificándose de tal modo que se ofertan por internet una variedad de productos que van desde alimentos clásicos: arándanos frescos, pulpa, mermelada, salsas, jugos, néctares, fruta confitada, congelada, así como arándanos en almíbar, helados, dulces; en mezclas con otros grupos alimenticios: yogurt con frutos del bosque, helados, productos de repostería, chocolate con arándanos, etc. Sin excluir a productos o suplementos dietéticos, cosméticos y productos de fitoterapia.

En este breve análisis de la especie emparentada con el mortiño, este fruto presenta la ventaja de que se puede congelar sin que se alteren sus características organolépticas y nutricionales (Cartilla, 2009), ni que ocurran variaciones en su peso o volumen; esto facilita su almacenamiento para mantener una oferta permanente aun fuera de las épocas de cosecha, así como la elaboración, diversificación, desarrollo e innovación de productos a partir del mortiño fresco y congelado.

\section{Conclusiones}

El mortiño (Vaccinium floribundum), es un arbusto de origen andino, crece de manera silvestre en los páramos del norte de la sierra andina, que en un amplio rango altitudinal desde los 1600 hasta los 3800 msnm; se desarrolla en climas templados y fríos, con temperaturas de 8 a $16^{\circ} \mathrm{C}$, lo que indica una marcada vegetación proveniente de los bosques seco montano bajo y húmedo montano.

Partiendo de los análisis bromatológicos realizados en el Departamento de Nutrición y Calidad del 
INIAP y análisis desarrollados por la FDA se determinó que el mortiño por su alto contenido de vitamina C (106,1 mg/100 g) y la presencia de antocianinas $(5 \% / 100 \mathrm{~g})$, es un indicador de su funcionalidad como antioxidante celular. Por su sabor agradable y cítrico fue un fruto muy apetecido en la dieta de nuestras etnias, los registros de etnomedicina resaltan su importancia medicinal por sus propiedades curativas a nivel del sistema gastrointestinal y sanguíneo.

El mortiño presenta varias posibilidades agroindustriales, considerando sus diversos usos ya sean culinarios (pastelería, vinos, helados y la tradicional colada morada) o sus usos industriales empleados en la fabricación de tintes, tinturas y combustibles. Este arbusto tiene vital importancia por su actividad regeneradora de sitios quemados, la cual se emplea en la reforestación de los páramos, contribuye a la protección de aves silvestres, suelos agrícolas y fuentes de agua.

\section{Referencias}

Aguilar, Z. 2009. Guía de plantas útiles de los páramos de Zuleta, Ecuador. EcoCiencia, Proyecto Páramo Andino. Programa de Apoyo a la Gestión Descentralizada de los Recursos Naturales en las Tres Provincias del Norte del Ecuador, pág. 99.

Alonso, J. 2004. Tratado de Fitofármacos y Nutracéuticos. Corpus Libros, Rosario-Argentina, primera edición edición, pág. 1360.

Asturizaga, A., B. Ollagaard y H. Balslev. 2006. Frutos comestibles. Botánica Económica de los Andes Centrales, págs. 329-346.

Badui, S. 2006. Química de los Alimentos. Pearson Educación, México, pág. 716.

Belleoud, L., D. Leluan y Boyer. 1967. Study on effects of anthocyanin glucosides on the nocturnal vision of air traffic controllers. Rev. Méd. Aéronaut, Spatial, 6: 5 .

Bernal, H. y J. Correa. 1990. Especies vegetales promisorias de los países del Convenio Andrés Be1lo, tomo VII. SECAB Ciencia y tecnología, Bogotá - Colombia, pág. 489.

Cartilla. 2009. Conozcamos y usemos el mortiño. Corporación Autónoma Regional del Centro de
Antioquia, Medellín, Corantioquia, tercera edición, pág. 14.

CESA. 1993. Usos tradicionales de las especies forestales nativas en el Ecuador, tomo 3. Central Ecuatoriana de Servicios Agrícolas, Quito - Ecuador, primera edición, pág. 258.

Cieza De León, P. 1962. Crónica del Perú. Espasa Calpe, Madrid - España, tercera edición, pág. 249.

Enandi, M. 1987. Relazione dell'esperto sulla documentazione farmacologica e tossicologica relativa alla specialita tegens (n.r.). Invernide la Beffa S.p.A.

Estrella, E. 1986. El pan de América: etnohistoria de los alimentos aborígenes en el Ecuador, tomo 29. Publicaciones del C.S.I.C. conmemorativas del V centenario del descubrimiento de América. Consejo Superior de Investigaciones Científicas, tercera edición, pág. 390.

Europea, C. E. 2009. Números E colorantes alimentarios. URL 〈http://europa.eu /legislation_summaries/other/121068a_es.htm $\rangle$.

Freire, F. A. 2004. Botánica sistemática ecuatoriana. Missouri Botanical Garden, pág. 209.

Fuentes, C. 2008. Estudio del mortiño y propuesta gastronómica aplicada a un recetario.

Gaviria, C., J. D. Hernández, M. Lobo, C. I. Medina y B. A. Rojano. 2012. Cambios en la actividad antioxidante en frutos de mortiño (vaccinium meridionale sw.) durante el desarrollo y maduración. Revista de la facultad de agronomía Medellin, 65: 6487-6495.

Gentry, A. H. 1988. Changes in plant community diversity and floristic composition on enveroinmental and geographic gradients. Ann. Missouri Botanical Garden, 75: 1-34.

Grismondi, G. 1980. Minerva ginecology. 32: 1-6.

INIAP. 2006. Informe del análisis bromatológico realizado en el departamento de nutrición y calidad del instituto nacional de investigaciones agropecuarias.

Jayle, G., M. Aubry, H. Gavini, G. Braccini y C. D. L. B. C. 1965. The action of anthocyanin glucosides on the scoptopic and mesopic vision of the normal subject. annals ocular. págs. 198-556. 
Jorgensen, P. M. y S. León-Yánez. 1999. Catalogue of vascular plants of ecuador. Syst. Bot. Missouri Bot. Gard, 75: 1-1181.

Jorgensen, P. M. C., R. Ulloa, Valencia y J. E. Madsen. 1995. A floristic analysis of the high andes of ecuador. S.P. Churchill et al. (editors), Biodiversity and Conservation of Neotropical Montane Forest. The New York Botanical Garden, págs. 221-237.

Kaddar, A., L. Robert, M. Miskulin, J. Tixier, D. Brechemier y A. Robert. 1979. Arterial wall. págs. 5187.

Kron, K. A., E. A. Powell y J. L. Luteyn. 2002. Phylogenetic relationships within the blueberry tribe (vaccinieae, ericaceae) based on sequence data from matk and nuclear ribosomal its regions, with comments on the placement of satyria. Amer. J. Bot., 89: 327-336.

León, P. L. D. 1962. Crónica del Perú (1550). Espasa Calpe, Madrid-España, tercera edición, pág. 294.

Loján, L. 2003. El verdor de los andes ecuatorianos: realidades y promesas. Proyecto Desarrollo Forestal Participativo en los Andes, pág. 296.

Luteyn, J. L. 2002. Diversity, adaptation, and endemism in neotropical Ericaceae: biogeographical patterns in the Vaccinieae, tomo 68. The Botanical Review, págs. 55-87.

Luteyn, J. L. y D. Silva. 1999. Hotspot for neotropical blueberry (ericaceae :vaccinieae). Murri (Antioquia Deparment, Colombia): Brittonia, 51: 280302.

MAGAP Ministerio de agricultura, a. y. p. 1998. Hoja técnica de mortiño-blueberry. Quito-Ecuador.

Mian, E., B. Curri, A. Lietti y E. Bombardelli. 1977. Minerva medicine. págs. 68-3565.

Morales, A. 2002-2011. Frutoterapia, nutrición y salud Plus Vitae. EDAF del Plata, Madrid-España, primera edición, pág. 212.

Noboa, V. 2010. Efecto de seis tipos de sustratos y tres dosis de ácido naftalenacético en la propagación vegetativa de mortiño (Vaccinium floribundum). Escuela Superior Politécnica de Chimborazo. Facultad de Recursos Naturales Escuela de Ingeniería Forestal, Riobamba-Ecuador.

Oviedo, Carrillo y G. Pacheco. 1992. Fundación natura. págs. 11, 37, 59, 89,103.

LA GRANJA, Revista de Ciencias de la Vida, 16(2) 2012: 5-13.

(C) 2012, Universidad Politécnica Salesiana, Ecuador.
Quingalombo, B. 2010. Investigación de productos autóctonos del Cantón Mejía (uvilla, mortiño) para su utilización dentro de la repostería. Universidad Tecnológica Equinoccial. Facultad de Turismo y Preservación Ambiental, Hotelería y Turismo, Quito-Ecuador.

Ramírez, M. y D. Williams. 2003. Guía agroculinaria de Cotacachi, Ecuador y alrededores. IPGRI-Américas.

Romero, R. 1991. Frutas silvestres de Colombia. Instituto de Cultura Hispánica, Bogotá, Segunda edición.

Ríos, M., M. J. Koziol, H. B. Pedersen y G. Granda. 2007. Plantas útiles del Ecuador: publicaciones, retos y perspectivas. Abya-Yala, Quito, Ecuador, pág. 652.

Salvayre, R., P. Braquet, T. Perruchot y L. DousteBlazy. 1982. Flavonoids and Bioflavonoids. Elsevier, Amsterdam, Oxford, New York, págs. 437442.

Smith, N., S. A. Mori, A. Henderson, D. W. Stevenson y S. V. Heald. 2004. Flowering plants of the neotropicas. Princeton University Press.

Torres, M. L. y D. Trujillo. 2010. Cultivo in vitro del mortiño (Vaccinium floribundum), tomo 2. Laboratorio de Biotecnología Vegetal, Universidad San Francisco de Quito, Quito, Ecuador, págs. B9-B15.

USDA. 2010. National nutrient database for standard reference. URL $\langle$ http://ndb.nal.usda.gov $/ \mathrm{ndb} /$ foods $/$ show $/ 2184$ ?fg=\&man=\&lfacet= \&count $=\& m a x=\&$ sort $=\& q$ lookup $=\&$ offset $=$ $\&$ format $=$ Stats\&new $=\rangle$.

Vasco, C. 2009. Phenolic compounds in ecuadorian fruits. Tesis Doctoral, Faculty of Natural Resources and Agricultural Sciences Department of Food Science. Uppsala. Doctoral Thesis. Swedish University of Agricultural Sciences.

Vasco, C., K. Rihinen, J. Ruales y A. Kamal-Eldin. 2009. Chemical composition and phenolic compound profile of mortiño (vaccinium floribundum). J. Agric. Food Chem, 57: 8274-8281.

Vavilov, N. I. 1951. Estudio sobre el origen de las plantas cultivadas. ACME Agency, pág. 185.

Zavarise, G. 1968. The effect of extended treatment with anthocyanosides on light sensitivity. Annali Oftalmolog. Clin. Oculary, 94: 209. 